\title{
AN APPLICATION ON DIFFERENTIAL EQUATIONS OF ORDER $m$
}

\author{
OSMAN ALTINTAŞ AND ÖZNUR ÖZKAN KILIÇ
}

Received 16 February, 2017

\begin{abstract}
In this paper we introduce the classes $\mathcal{T}_{n}(p, \lambda, A, B)$ and $\mathcal{K}_{n}(p, \lambda, \mu, m, A, B)$ and derive distortion inequalities of the functions belonging to class $\mathcal{K}_{a}(p, \lambda, \mu, m, A, B)$. Further we apply to the $(n, \delta)$ - neighborhoods of functios in the class $\mathcal{K}_{n}(p, \lambda, \mu, m, A, B)$.
\end{abstract}

2010 Mathematics Subject Classification: 30C45

Keywords: analytic function, multivalent function, subordination, $(n, \delta)-$ neighborhoods, coefficient bounds, distortion inequalities

\section{INTRODUCTION AND DEFINITIONS}

Let $\mathcal{T}_{n}(p)$ denote the class of functions $f(z)$ normalized by

$$
f(z)=z^{p}-\sum_{k=n+p}^{\infty} a_{k} z^{k} \quad\left(a_{k} \geq 0 ; n, p \in \mathbb{N}:=\{1,2,3, \cdots\}\right)
$$

which are analytic and p-valent in the open unit disk $\mathbb{U}=\{z \in \mathbb{C}:|z|<1\}$ on the complex plane $\mathbb{C}$.

Let $f$ and $F$ be analytic functions in the unit disk $\mathbb{U}$. A function $f$ is said to be subordinate to $F$, written as $f \prec F$ or $f(z) \prec F(z)$, if there exists a Schwarz function $\omega: \mathbb{U} \rightarrow \mathbb{U}$ with $\omega(0)=0$ such that $f(z)=F(\omega(z))$. In particular, if $F$ is univalent in $\mathbb{U}$, we have the following equivalence:

$$
f(z) \prec F(z) \Longleftrightarrow[f(0)=F(0) \wedge f(\mathbb{U}) \subseteq F(\mathbb{U})] .
$$

Following the earlier investigations by Goodman [11] and Ruscheweyh [15] (see also $[1-3,5,6,9,13])$, we define the $(n, \delta)$-neighborhoods of functions $f \in \mathcal{T}_{n}(p)$ by

$$
\mathcal{N}_{n, \delta}(f ; g)=\left\{g \in \mathcal{T}_{n}(p): g(z)=z^{p}-\sum_{k=n+p}^{\infty} b_{k} z^{k} \text { and } \sum_{k=n+p}^{\infty} k\left|a_{k}-b_{k}\right| \leq \delta\right\} .
$$

Let $\mathcal{S}^{*}$ and $\mathcal{C}$ be the usual subclasses of functions which members are univalent, starlike and convex in $\mathbb{U}$, respectively. 
A function $f \in \mathcal{T}_{n}(p)$ is called p-valently starlike of order $\gamma$ if it satisfies the conditions

$$
\Re\left(\frac{z f^{\prime}(z)}{f(z)}\right)>\gamma
$$

and

$$
\int_{0}^{2 \pi} \Re\left(\frac{z f^{\prime}(z)}{f(z)}\right) d \theta=2 p \pi
$$

for $0 \leq \gamma<p, p \in \mathbb{N}$ and $z \in \mathbb{U}$. We denote by $\mathcal{S}_{n}^{*}(p, \gamma)$ the class of all $\mathrm{p}$-valently starlike functions of order $\gamma$. Furthermore, a function $f \in \mathcal{T}_{n}(p)$ is called p-valently convex of order $\gamma$ if it satisfies the conditions

$$
\Re\left(1+\frac{z f^{\prime \prime}(z)}{f^{\prime}(z)}\right)>\gamma
$$

and

$$
\int_{0}^{2 \pi} \Re\left(1+\frac{z f^{\prime \prime}(z)}{f^{\prime}(z)}\right) d \theta=2 p \pi
$$

for $0 \leq \gamma<p, p \in \mathbb{N}$ and $z \in \mathbb{U}$. We denote by $\mathcal{C}_{n}(p, \gamma)$ the class of all p-valently convex functions of order $\gamma$.

Clearly, $\mathcal{S}^{*}:=\mathcal{S}_{1}^{*}(1,0)$ and $\mathcal{C}:=\mathcal{C}_{1}(1,0)$. We note that

$$
f(z) \in \mathcal{C}_{n}(p, \gamma) \Leftrightarrow \frac{f^{\prime}(z)}{p} \in \mathcal{S}_{n}^{*}(p, \gamma)
$$

The classes $\mathcal{S}_{n}^{*}(p, \gamma)$ and $\mathcal{C}_{n}(p, \gamma)$ were introduced by Patil and Thakare [14].

Therefore, various subclasses of $p$-valent functions in $\mathbb{U}$ was studied by Altıntaş et al. in [8], Nunokawa et al. in [12] and Srivastava et al. in [16,17].

A function $f \in \mathcal{T}_{n}(p)$ is called Janowski p-valently starlike if it satisfies the condition

$$
\frac{z f^{\prime}(z)}{f(z)} \prec p \frac{1+A z}{1+B z}
$$

for $-1 \leq A<B \leq 1, p \in \mathbb{N}$ and $z \in \mathbb{U}$. We denote by $\mathcal{S}_{n}^{*}(p, A, B)$ the class of all Janowski p-valently starlike functions.

Also, a function $f \in \mathcal{T}_{n}(p)$ is called Janowski p-valently convex if it satisfies the condition

$$
1+\frac{z f^{\prime \prime}(z)}{f^{\prime}(z)} \prec p \frac{1+A z}{1+B z}
$$

for $-1 \leq A<B \leq 1, p \in \mathbb{N}$ and $z \in \mathbb{U}$. We denote by $\mathcal{C}_{n}(p, A, B)$ the class of all Janowski p-valently convex functions.

We note that, $\mathcal{S}_{n}^{*}(p, \gamma):=\mathcal{S}_{n}^{*}(p, 1-2 \gamma,-1), \mathcal{S}^{*}:=\mathcal{S}_{1}^{*}(1,1,-1)$ and $\mathcal{C}_{n}(p, \gamma):=$ $\mathcal{C}_{n}(p, 1-2 \gamma,-1), \mathcal{C}:=\mathcal{C}_{1}(1,1,-1)$. 
Let $\mathcal{T}_{n}(p, \lambda, A, B)$ denote the subclass of $\mathcal{T}_{n}(p)$ consisting of functions $f(z)$ which satisfy the following inequality:

$$
\frac{z f^{\prime}(z)+\lambda z^{2} f^{\prime \prime}(z)}{\lambda z f^{\prime}(z)+(1-\lambda) f(z)} \prec p \frac{1+A z}{1+B z}
$$

where $0 \leq \lambda \leq 1,-1 \leq A<B \leq 1, p \in \mathbb{N}, z \in \mathbb{U}$. The class $\mathcal{T}_{n}(p, \lambda, A, B)$ was introduced and studied by Altıntaş in [3,7].

Clearly, we have the following relationships:

$$
\mathcal{S}_{n}^{*}(p, A, B):=\mathcal{T}_{n}(p, 0, A, B) \text { and } \mathcal{C}_{n}(p, A, B):=\mathcal{T}_{n}(p, 1, A, B) .
$$

We note that these classes are studied in [10].

Recently, we have defined and studied in $[1,2,4-6]$ the following second order differential equation:

$$
z^{2} \frac{d^{2} w}{d z^{2}}+2(\mu+1) z \frac{d w}{d z}+\mu(\mu+1) w=(p+\mu)(p+\mu+1) g
$$

where $w=f(z) \in \mathcal{T}_{n}(p), g=g(z)$ satisfy the following inequality:

$$
\Re \frac{z g^{\prime}(z)+\lambda z^{2} g^{\prime \prime}(z)}{\lambda z g^{\prime}(z)+(1-\lambda) g(z)}>\alpha
$$

where $0 \leq \lambda \leq 1,0 \leq \alpha<1, p \in \mathbb{N}, \mu>-p, z \in \mathbb{U}$.

Definition 1. The following non-homogenous Cauchy-Euler differential equation of order 3 is

$$
\begin{aligned}
z^{3} \frac{d^{3} w}{d z^{3}}+3(\mu+2) z^{2} \frac{d^{2} w}{d z^{2}} & +3(\mu+1)(\mu+2) z \frac{d w}{d z}+\mu(\mu+1)(\mu+2) w \\
= & (p+\mu)(p+\mu+1)(p+\mu+2) g
\end{aligned}
$$

where $w=f(z) \in \mathcal{T}_{n}(p), g=g(z) \in \mathcal{T}_{n}(p, \lambda, A, B)$ and $\mu>-p$.

This differential equation is defined and studied in [3].

Definition 2. The following non-homogenous Cauchy-Euler differential equation of order $\mathrm{m}$ is

$$
\begin{gathered}
z^{m} \frac{d^{m} w}{d z^{m}}+\left(\begin{array}{c}
m \\
1
\end{array}\right)(\mu+m-1) z^{m-1} \frac{d^{m-1} w}{d z^{m-1}}+\ldots+\left(\begin{array}{c}
m \\
r
\end{array}\right) \prod_{j=r}^{m-1}(\mu+j) z^{r} \frac{d^{r} w}{d z^{r}}+ \\
\ldots+\left(\begin{array}{c}
m \\
m
\end{array}\right) \prod_{j=0}^{m-1}(\mu+j) w=\prod_{j=0}^{m-1}(p+\mu+j) g
\end{gathered}
$$

where $w=f(z) \in \mathcal{T}_{n}(p), g=g(z) \in \mathcal{T}_{n}(p, \lambda, A, B), m \in \mathbb{N}^{*}:=\{2,3, \cdots\}$ and $\mu>-p$.

Finally $\mathcal{K}_{n}(p, \lambda, \mu, m, A, B)$ denote the subclass of the class $\mathcal{T}_{n}(p)$ consisting of functions $f(z)$, satisfying the equation (1.14) in Definition 2.

In this paper, we obtain coefficient bounds, distortion inequalities and $(n, \delta)-$ neighborhoods of functions $f \in \mathcal{T}_{n}(p)$ in the class $\mathcal{K}_{n}(p, \lambda, \mu, m, A, B)$. 


\section{COEFFICIENT Bounds AND Distortion INEQualities}

For proving the main results in this paper, we will use the following lemmas.

Lemma 1 ([3]). Let the function $\mathcal{T}_{n}(p)$ be defined by (1.1). Then $f(z)$ is in the class $\mathcal{T}_{n}(p, \lambda, A, B)$ if and only if

$$
\sum_{k=n+p}^{\infty}(k-p-p A+k B)(\lambda k-\lambda+1) a_{k} \leq p(B-A)(\lambda p-\lambda+1)
$$

where $0 \leq \lambda \leq 1,-1 \leq A<B \leq 1, p \in \mathbb{N}$.

The result is sharp for the function $f(z)$ given by

$$
f(z)=z^{p}-\frac{p(B-A)(\lambda p-\lambda+1)}{[(n+p)(1+B)-p(1+A)][\lambda(n+p)-\lambda+1]} z^{n+p} .
$$

Lemma 2 ([3]). Let the function $f(z) \in \mathcal{T}_{n}(p)$ defined by (1.1) be in the class $\mathcal{T}_{n}(p, \lambda, A, B)$. Then, we have

$$
\sum_{k=n+p}^{\infty} a_{k} \leq \frac{p(B-A)(\lambda p-\lambda+1)}{[(n+p)(1+B)-p(1+A)][\lambda(n+p)-\lambda+1]}
$$

and

$$
\sum_{k=n+p}^{\infty} k a_{k} \leq \frac{p(B-A)(\lambda p-\lambda+1)(n+p)}{[(n+p)(1+B)-p(1+A)][\lambda(n+p)-\lambda+1]} .
$$

The distortion inequalities for functions in the class $\mathcal{K}_{a}(p, \lambda, \mu, m, A, B)$ are given by Theorem 1 below.

Theorem 1. If a function $f \in \mathcal{T}_{n}(p)$ is in the class $\mathcal{K}_{n}(p, \lambda, \mu, m, A, B)$, then

$$
\begin{aligned}
& |f(z)| \leq|z|^{p}+ \\
& \frac{p(B-A)(\lambda p-\lambda+1) \prod_{j=0}^{m-1}(p+\mu+j)}{(m-1)[(n+p)(1+B)-p(1+A)][\lambda(n+p)-\lambda+1] \prod_{j=0}^{m-2}(n+p+\mu+j)}|z|^{n+p}
\end{aligned}
$$

and

$$
\begin{aligned}
& |f(z)| \geq|z|^{p}- \\
& \frac{p(B-A)(\lambda p-\lambda+1) \prod_{j=0}^{m-1}(p+\mu+j)}{(m-1)[(n+p)(1+B)-p(1+A)][\lambda(n+p)-\lambda+1] \prod_{j=0}^{m-2}(n+p+\mu+j)}|z|^{n+p} .
\end{aligned}
$$

Proof. We first suppose that a function $f \in \mathcal{T}_{n}(p)$ is in the class $\mathcal{K}_{n}(p, \lambda, \mu, m, A, B)$. Let the function $g(z)=z^{p}-\sum_{k=n+p}^{\infty} b_{k} z^{k} \in \mathcal{T}_{n}(p, \lambda, A, B)$ occurring in the non-homogenous Cauchy-Euler differential equation of order $m$ in (1.14) with, of course,

$$
b_{k} \geq 0 \quad(k=n+p, n+p+1, \ldots) .
$$


Then, we readily find from (1.14) that

$$
a_{k}=\frac{\prod_{j=0}^{m-1}(p+\mu+j)}{\prod_{j=0}^{m-1}(k+\mu+j)} b_{k} \quad(k=n+p, n+p+1, \ldots) .
$$

so that

$$
f(z)=z^{p}-\sum_{k=n+p}^{\infty} \frac{\prod_{j=0}^{m-1}(p+\mu+j)}{\prod_{j=0}^{m-1}(k+\mu+j)} b_{k} z^{k} .
$$

Since $g \in \mathcal{T}_{n}(p, \lambda, A, B)$, the first assertion (2.3) of Lemma 2 yields the following inequality:

$$
b_{k} \leq \frac{p(B-A)(\lambda p-\lambda+1)}{[(n+p)(1+B)-p(1+A)][\lambda(n+p)-\lambda+1]} .
$$

Together with (2.8) and (2.9) yields that

$$
\begin{aligned}
& |f(z)| \leq|z|^{p}+ \\
& |z|^{n+p} \frac{p(B-A)(\lambda p-\lambda+1)}{[(n+p)(1+B)-p(1+A)][\lambda(n+p)-\lambda+1]} \sum_{k=n+p}^{\infty} \frac{\prod_{j=0}^{m-1}(p+\mu+j)}{\prod_{j=0}^{m-1}(k+\mu+j)}
\end{aligned}
$$

and using the following identity that

$$
\begin{aligned}
& \sum_{k=n+p}^{\infty} \frac{1}{\prod_{j=0}^{m-1}(k+\mu+j)} \\
& =\frac{1}{(m-1) !} \sum_{k=n+p}^{\infty}\left[\frac{\left(\begin{array}{c}
m-1 \\
0
\end{array}\right)}{k+\mu}-\frac{\left(\begin{array}{c}
m-1 \\
1
\end{array}\right)}{k+\mu+1}+\cdots+(-1)^{m-1} \frac{\left(\begin{array}{c}
m-1 \\
k+1
\end{array}\right)}{k+m-1}\right] \\
& =\frac{1}{m-1} \frac{1}{\prod_{j=0}^{m-2}(n+p+\mu+j)}
\end{aligned}
$$

where $\mu \in \mathbb{R} \backslash\{-n-p,-n-p-1, \ldots\}$. The assertion (2.5) of Theorem 1 follows at once from (2.10) with (2.11). The assertion (2.6) of Theorem 1 can be proven by similarly.

Corollary 1 ([3]). If $f \in \mathcal{K}_{a}(p, \lambda, \mu, 2, A, B)$, then we have

$$
|f(z)| \leq|z|^{p}+\frac{p(B-A)(\lambda p-\lambda+1)(p+\mu)(p+\mu+1)}{[(n+p)(1+B)-p(1+A)][\lambda(n+p)-\lambda+1](n+p+\mu)}|z|^{n+p}
$$

and

$$
|f(z)| \geq|z|^{p}-\frac{p(B-A)(\lambda p-\lambda+1)(p+\mu)(p+\mu+1)}{[(n+p)(1+B)-p(1+A)][\lambda(n+p)-\lambda+1](n+p+\mu)}|z|^{n+p} .
$$


Corollary 2. If $f \in K_{n}(p, \lambda, \mu, 3, A, B)$, then we have

$$
\begin{aligned}
& |f(z)| \leq|z|^{p}+ \\
& \frac{p(B-A)(\lambda p-\lambda+1)(p+\mu)(p+\mu+1)(p+\mu+2)}{2[(n+p)(1+B)-p(1+A)][\lambda(n+p)-\lambda+1](n+p+\mu)(n+p+\mu+1)}|z|^{n+p} \\
& |f(z)| \geq|z|^{p}- \\
& \frac{p(B-A)(\lambda p-\lambda+1)(p+\mu)(p+\mu+1)(p+\mu+2)}{2[(n+p)(1+B)-p(1+A)][\lambda(n+p)-\lambda+1](n+p+\mu)(n+p+\mu+1)}|z|^{n+p} .
\end{aligned}
$$

and

3. NeIghborhoods For The Class $\mathcal{K}_{n}(p, \lambda, \mu, m, A, B)$

In this section, we determine inclusion relations for the class $\mathcal{K}_{n}(p, \lambda, \mu, m, A, B)$ concerning the $(n, \delta)$-neighborhoods defined by (1.2).

Theorem 2. If $f \in \mathcal{T}_{n}(p)$ is in the class $\mathcal{K}_{n}(p, \lambda, \mu, m, A, B)$, then

$$
\mathcal{K}_{b}(p, \lambda, \mu, m, A, B) \subset N_{n, \delta}(g ; f)
$$

where $g(z)$ is given by (1.14) and

$$
\begin{aligned}
\delta:=\frac{p(B-A)(\lambda p-\lambda+1)(n+p)}{[(n+p)(1+B)-p(1+A)][\lambda(n+p)-\lambda+1]} \\
{\left[1+\frac{\prod_{j=0}^{m-1}(p+\mu+j)}{(m-1) \prod_{j=0}^{m-2}(n+p+\mu+j)}\right] . }
\end{aligned}
$$

Proof. Suppose that $\mathcal{K}_{n}(p, \lambda, \mu, m, A, B)$. Then, upon substituting from (2.7) into the following coefficient inequality:

$$
\sum_{k=n+p}^{\infty} k\left|b_{k}-a_{k}\right| \leq \sum_{k=n+p}^{\infty} k b_{k}+\sum_{k=n+p}^{\infty} k a_{k} \quad\left(a_{k} \geq 0, b_{k} \geq 0\right)
$$

we obtain that

$$
\sum_{k=n+p}^{\infty} k\left|b_{k}-a_{k}\right| \leq \sum_{k=n+p}^{\infty} k b_{k}+\sum_{k=n+p}^{\infty} \frac{\prod_{j=0}^{m-1}(p+\mu+j)}{\prod_{j=0}^{m-1}(k+\mu+j)} k b_{k} .
$$

Since $g \in \mathcal{T}_{n}(p, \lambda, A, B)$, the second assertion (2.4) of Lemma 2 yields that

$$
k b_{k} \leq \frac{p(B-A)(\lambda p-\lambda+1)(n+p)}{[(n+p)(1+B)-p(1+A)][\lambda(n+p)-\lambda+1]}(k=n+p, n+p+1, \ldots) .
$$

In the right hand side of (3.4), we obtain the assertion (3.2) using (3.5) and (2.11), respectively.

Thus, by Definition 2 with $g(z)$ interchanged by $f(z)$, we conclude that

$$
f \in N_{n, \delta}(g ; f) \text {. }
$$


This completes the proof of Theorem 2 .

Corollary 3 ([3]). If $f \in \mathcal{K}_{n}(p, \lambda, \mu, 2, A, B)$, then

$$
\mathcal{K}_{a}(p, \lambda, \mu, 2, A, B) \subset N_{n, \delta}(g ; f)
$$

where $g(z)$ is given by (1.14) for $m=2$ and $\delta$ is given by

$$
\delta:=\frac{p(B-A)(\lambda p-\lambda+1)(n+p)}{[(n+p)(1+B)-p(1+A)][\lambda(n+p)-\lambda+1]}\left[1+\frac{(p+\mu)(p+\mu+1)}{n+p+\mu}\right] .
$$

Corollary 4. If $f \in \mathcal{K}_{n}(p, \lambda, \mu, 3, A, B)$, then

$$
\mathcal{K}_{a}(p, \lambda, \mu, 3, A, B) \subset N_{n, \delta}(g ; f)
$$

where $g(z)$ is given by (1.14) for $m=3$ and $\delta$ is given by

$$
\begin{aligned}
\delta:=\frac{p(B-A)(\lambda p-\lambda+1)(n+p)}{[(n+p)(1+B)-p(1+A)][\lambda(n+p)-\lambda+1]} \\
{\left[1+\frac{(p+\mu)(p+\mu+1)(p+\mu+2)}{2(n+p+\mu)(n+p+\mu+1)}\right] . }
\end{aligned}
$$

\section{REFERENCES}

[1] O. Altintaş, O. Özkan, and H. M. Srivastava, "Neighborhoods of a certain family of multivalent functions with negative coefficients." Comput. Math. Appl., vol. 47, no. 10-11, pp. 1667-1672, 2004, doi: 10.1016/j.camwa.2004.06.014.

[2] O. Altıntaş, "Neighborhoods of certain $p$-valently analytic functions with negative coefficients." Appl. Math. Comput., vol. 187, no. 1, pp. 47-53, 2007, doi: 10.1016/j.amc.2006.08.101.

[3] O. Altıntaş, "Certain applications of subordination associated with neighborhoods." Hacet. J. Math. Stat., vol. 39, no. 4, pp. 527-534, 2010.

[4] O. Altıntaş, H. Irmak, S. Owa, and H. M. Srivastava, "Coefficient bounds for some families of starlike and convex functions of complex order." Appl. Math. Lett., vol. 20, no. 12, pp. 1218 1222, 2007, doi: 10.1016/j.aml.2007.01.003.

[5] O. Altıntaş, H. Irmak, and H. M. Srivastava, "Neighborhoods for certain subclasses of multivalently analytic functions defined by using a differential operator." Comput. Math. Appl., vol. 55, no. 3, pp. 331-338, 2008, doi: 10.1016/j.camwa.2007.03.017.

[6] O. Altintaş, O. Özkan, and H. M. Srivastava, "Neighborhoods of a class of analytic functions with negative coefficients." Appl. Math. Lett., vol. 13, no. 3, pp. 63-67, 2000, doi: 10.1016/S08939659(99)00187-1.

[7] O. Altintaş, O. Özkan, and H. M. Srivastava, "Majorization by starlike functions of complex order." Complex Variables, Theory Appl., vol. 46, no. 3, pp. 207-218, 2001, doi: 10.1080/17476930108815409.

[8] O. Altintaş and H. M. Srivastava, "Some majorization problems associated with $p$-valently starlike and convex functions of complex order." EAMJ, East Asian Math. J., vol. 17, no. 2, pp. 175-183, 2001.

[9] O. Altintas and S. Owa, "Neighborhoods of certain analytic functions with negative coefficients." Int. J. Math. Math. Sci., vol. 19, no. 4, pp. 797-800, 1996, doi: 10.1155/S016117129600110X.

[10] R. Goel and N. Sohi, "Multivalent functions with negative coefficients." Indian J. Pure Appl. Math., vol. 12, pp. 844-853, 1981.

[11] A. W. Goodman, Univalent functions. Tampa, FL: Mariner Publishing Co., 1983, vol. I. 
[12] N. Nunokawa, H. M. Srivastava, N. Tuneski, and B. Jolevska-Tuneska, "Some Marx-Strohhäcker type results for a class of multivalent functions." Miskolc Math. Notes, vol. 18, no. 1, pp. 353-364, 2017, doi: 10.18514/MMN.2017.1952.

[13] O. Özkan and O. Altintas, "On neighborhoods of a certain class of complex order defined by Ruscheweyh derivative operator.” JIPAM, J. Inequal. Pure Appl. Math., vol. 7, no. 3, p. 7, 2006.

[14] D. Patil and N. Thakare, "On convex hulls and extreme points of p-valent starlike and convex classes with applications.” Bull. Math. Soc. Sci. Math. Répub. Soc. Roum., Nouv. Sér., vol. 27, pp. 145-160, 1983.

[15] S. Ruscheweyh, "Neighborhoods of univalent functions." Proc. Am. Math. Soc., vol. 81, pp. 521527, 1981, doi: 10.2307/2044151.

[16] H. M. Srivastava and S. Bulut, "Neighborhoods proprties of certain classes of multivalently analytic functions associated with the convolution structure." Appl. Math. Comput., vol. 218, no. 11, pp. 6511-6518, 2012, doi: 10.1016/j.amc.2011.12.022.

[17] H. M. Srivastava, R. M. El-Ashwah, and N. Breaz, "A certain subclass of multivalent functions involving higher-order derivatives." Filomat, vol. 30, no. 1, pp. 113-124, 2016, doi: 10.2298/FIL1601113S.

Authors' addresses

Osman Altıntas

Başkent University, Department of Mathematics Education, Bağlıca, 06810 Ankara, Turkey

E-mail address: oaltintas@baskent.edu.tr

Öznur Özkan Kılıç

Başkent University, Department of Technology and Knowledge Management, Bağlıca, TR 06790 Ankara, Turkey

E-mail address: oznur@baskent.edu.tr 